\title{
Design of a Prototype Readout Electronics for a Low-energy Ion Spectrometer in Near-earth Space
}

\section{Zhenyu Sun ${ }^{1}$}

CAS Key Laboratory of Geospace Environment, University of Science and Technology of China CAS Center for Excellence in Comparative Planetology, University of Science and Technology of China Mengcheng National Geophysical Observatory, University of Science and Technology of China Hefei, 230026, China

E-mail: szy2016@mail.ustc.edu.cn

\section{Zhe Cao*}

State Key Laboratory of Particle Detection and Electronics (IHEP-USTC)

Department of Modern Physics, University of Science and Technology of China,

CAS Center for Excellence in Comparative Planetology, University of Science and Technology of China Hefei, 230026, China

E-mail: caozhedustc.edu.cn

\section{Guangyuan Yuan}

State Key Laboratory of Particle Detection and Electronics (IHEP-USTC)

Department of Modern Physics, University of Science and Technology of China,

Hefei, 230026, China

E-mail: yuanyg@mail.ustc.edu.cn

\section{Shuwen Wang}

State Key Laboratory of Particle Detection and Electronics (IHEP-USTC)

Department of Modern Physics, University of Science and Technology of China,

Hefei, 230026, China

E-mail: wswemail.ustc.edu.cn

\section{Yiren Li}

CAS Key Laboratory of Geospace Environment, University of Science and Technology of China

CAS Center for Excellence in Comparative Planetology, University of Science and Technology of China Mengcheng National Geophysical Observatory, University of Science and Technology of China

Hefei, 230026, China

E-mail: Iyroustc.edu.cn

\section{Kai Liu}

CAS Key Laboratory of Geospace Environment, University of Science and Technology of China CAS Center for Excellence in Comparative Planetology, University of Science and Technology of China Mengcheng National Geophysical Observatory, University of Science and Technology of China

Hefei, 230026, China

E-mail: kailiudustc.edu.cn

\section{Shubin Liu}

State Key Laboratory of Particle Detection and Electronics (IHEP-USTC)

Department of Modern Physics, University of Science and Technology of China,

Hefei, 230026, China

E-mail: Iiushbaustc.edu.cn

\section{${ }^{1}$ Speaker; *Corresponding Author}




\title{
Qi An
}

State Key Laboratory of Particle Detection and Electronics (IHEP-USTC)

Department of Modern Physics, University of Science and Technology of China,

Hefei, 230026, China

E-mail: anqieustc.edu.cn

\begin{abstract}
A low-energy ion spectrometer has been successfully developed, with the objective of measuring low-energy ions of $100 \mathrm{eV} / \mathrm{e} \sim 25 \mathrm{keV} / \mathrm{e}$ on a three-axis stabilized satellite in geosynchronous orbit. This instrument requires that the readout electronics system has the characteristics of 16 micro-channel plates (MCP) channels for $22.5^{\circ}$ angle resolution detection, sensitivity higher than $1 \times 10^{4}$ electrons, event rate up to $10 \mathrm{MHz}$, and less than $10 \mathrm{~W}$ power consumption.

In this paper, radiation-hardened readout electronics has been designed for the detector, considering the space radiation environment. The system consists 16 low-noise charge sensitive preamplifier-discriminators (A121s) which are employed for data acquisition of MCP channels to use in fast pulse counting mode, antifuse field programmable gate array (FPGA) which is used for data storage and package, high voltage (HV) control, self-test circuits, states monitor module and communication module with upper host. In addition, what improve the reliability of the system is the FPGA-based satellite onboard software adopts the method of triple modular redundancy (TMR) and refreshes all external key registers at regular time.

During the performance experiments, the test results show that the readout electronics achieves $12 \mathrm{MHz}$ event rate, $5 \times 10^{4}$ electrons sensitivity, and total power consumption of $4.73 \mathrm{~W}$, showing that the performance meets the spectrometer physical requirement. The system stably operates all function in a series of environment tests.
\end{abstract}

Key words: Low-energy ion spectrometer, Readout electronics, Antifuse FPGA, A121

36th International Cosmic Ray Conference -ICRC2019.

July 24th - August 1st, 2019

Madison, WI, U.S.A. 


\section{Introduction}

Space plasma detection has been a focus of attention for space scientists since the end of 20th century. The research on space plasma is significant for the aerospace industry, radio communication, solar wind early warning, planetary atmosphere, etc. Several satellites have been launched for detecting space plasmas around the earth, such as Cluster [1] and AMPTE [2]. Their three-dimensional velocity distribution of low-energy ions depends on the spin of satellite. This way is difficult to accurately and comprehensively measure the properties of low-energy ions on a three-axis stabilized satellite. Therefore, an omni-directional low-energy ion spectrometer installed on a three-axis stabilized satellite has been successfully developed in geosynchronous orbit. The structural diagram of the spectrometer is shown in Fig. 1.

Two sweep HV ( $\mathrm{U}_{\text {sweep1 }}$ and $\mathrm{U}_{\text {sweep2 } 2}$ ), range from $-2800 \mathrm{~V}$ to $2800 \mathrm{~V}$, are applied to the entrance to choose ions from different directions. The entrance unit is implemented by a hemispherical top-hat electrostatic analyzer (ESA) [3]. A sweep HV $\left(\mathrm{U}_{\mathrm{ESA}}\right)$, range from -2800 V to $0 \mathrm{~V}$, is applied to the inner hemisphere for distinguishing incident ions with different energy. The passed ions are amplified by the MCP and then hit the anode board. The readout electronics system records the hit signals, analyses energy spectrum and provides HV and low voltage power supplies on a three-axis stabilized satellite.

This low-energy ion spectrometer focuses on ions with the energy between $100 \mathrm{eV}$ and 25 $\mathrm{keV}$. The field of view (FOV) of it is $360^{\circ}$, divided into 16 detection directions. The azimuth resolution of inlet deflector system is $22.5^{\circ}$. The pitch angle FOV is $90^{\circ}\left(-45^{\circ} \sim 45^{\circ}\right)$, divided into 8 detection directions. The elevation angle is $11.25^{\circ}$.

In this paper, the readout electronics to fulfill the requirements of spectrometer is introduced and test performance is described.

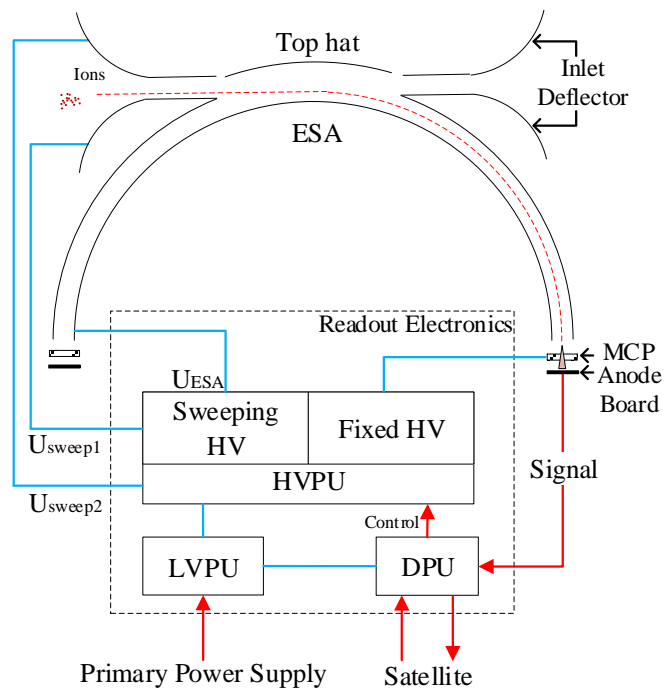

Fig. 1. Structural diagram of the spectrometer

\section{Electronics design}

According to principle of the spectrometer in Fig. 1, the main function of the readout electronics is to count signals at different energy steps controlled by the HV supplies. Then the real-time analysis of energy spectrum data is transmitted to the satellite for analysis.The details of readout electronics to satisfy the instrument requirements are as follows: 
1. Realizing 16 signal acquisition channels from 16 evenly distributed MCPs for $22.5^{\circ}$ angle resolution detection;

2. Design of hardware and software need to consider radiation resistance and reliability because of the harsh space environment in geosynchronous orbit;

3. Electronics system requires low data rates $(<10 \mathrm{kbps})$ and low power consumption $(<10 \mathrm{~W})$ for satellite mission;

4. Rapid stable sweep HV circuit with a maximum output of $\pm 2800 \mathrm{~V}$ due to the structure of inlet deflector system and the ESA.

Finally, the readout electronics design diagram is shown in Fig. 2, which is divided into three units: low voltage power unit (LVPU), high voltage power unit (HVPU) and data processing unit (DPU). The electronic box (E-box) is composed of three units.

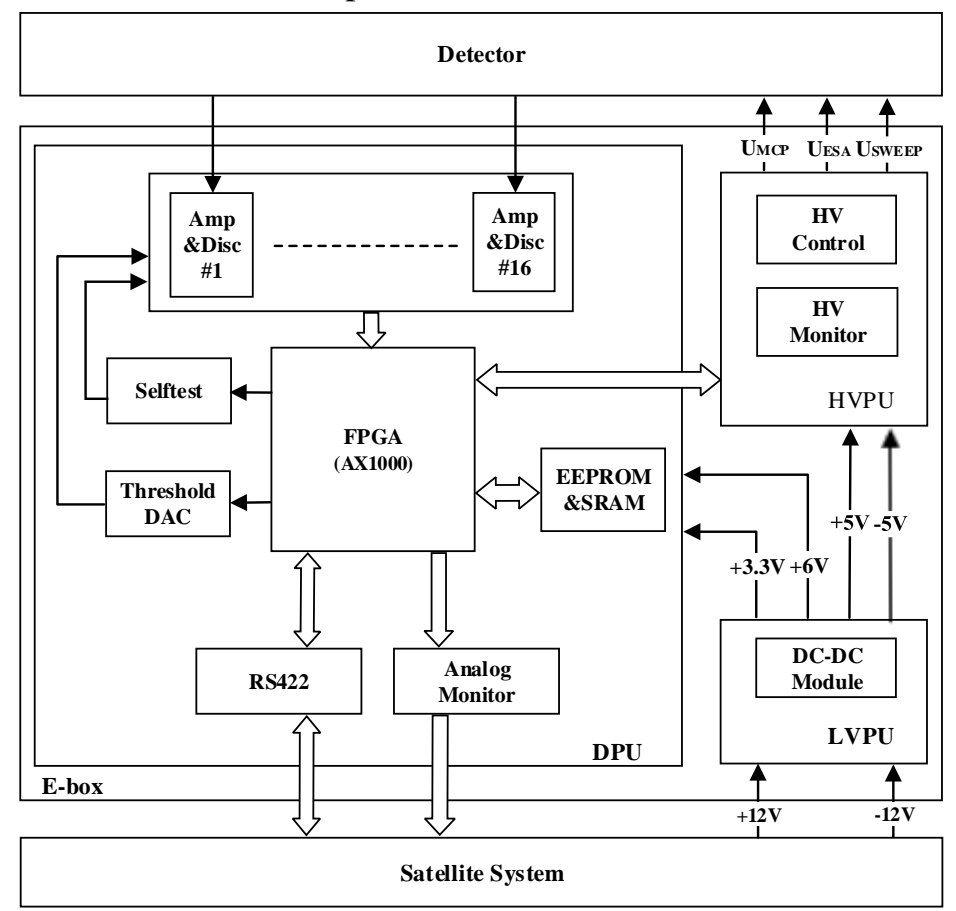

Fig. 2. Diagram of the readout electronics

\subsection{Hardware design}

\subsubsection{Signal acquisition and self-test circuit}

The signal acquisition circuit is to enlarge small hit signal from MCP, to screen the crossthreshold, and to count. Achieveing high event rate (>10 MHz) of ions in geosynchronous orbit and radiation-hardened are something the electronics system takes into consederation. Therefore, 16 low-noise charge sensitive preamplifier-discriminators (A121, Amptek) are employed for 16 $\mathrm{MCP}$ data acquisition channels for $22.5^{\circ}$ angle resolution detection in this project. The component was also used for the Jovian Auroral Distributions Experiment (JADE) on the Juno Mission to Jupiter [4]. Compared with the previous version A111/A111F, A121 has higher event rate (12 $\mathrm{MHz})$, higher electrons sensitivity $\left(5 \times 10^{4}\right)$ and less rise time [5]. The threshold can be adjusted easily by changing the voltage at threshold adjustment pin via the digital-to-analog converter (DAC), so that the remote control is more flexible. While the threshold of previous version can only be adjusted via changing the resistance of the associated circuit. 


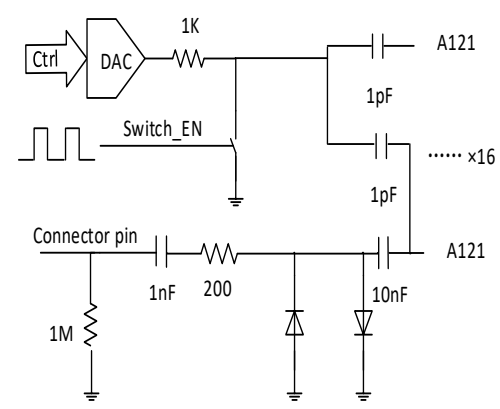

Fig. 3 Diagram of the detector signal input circuit and self-test circuit.

The signal input and Electrostatic Discharge (ESD) protecting circuits are shown in Fig. 3. In addition, a built-in self-test circuit is designed to calibrate the electronics. In the test mode, HV supply for detectors is turned off and the DAC is controlled by the FPGA to output adequate analog voltage. A switch enable signal, generated by the FPGA, will be applied to an analog switch to produce test pulse to trigger A121s by capacitive coupling. In the HV mode, the switch keeps closed in order to reduce noise, and A121s are triggered by detectors.

\subsubsection{FPGA control circuit}

The control circuit is the core of the electronics system. Counting statistics, HV control, data storage, states monitor and communication are its functions. The selection of FPGA chip as a control chip has the following characteristics: high integration, simple software development and low power consumption. Three kinds of radiation effects are taken into consideration, including the Total Ionizing Dose (TID) effect, the Single Event Upset (SEU) effect and the Single Event Latch-up (SEL) effect. Antifuse FPGA is the best anti-irradiation performance of all FPGAs, mainly used in the aerospace field. The antifuse FPGA (AX1000, Microsemi Corp.) is the core model in this system, which is immune to SEL.The configuration information of its logic is regarded to be demonstrated hardness against SEU and the TID is greater than $1 \mathrm{Mrad}(\mathrm{Si})$ [6].

Considering the reliability of communication and the requirement of system data rates (1920bps), the project employs a RS422 interface to send instructions and upload scientific data to the satellite per hour. A 4M bits Static Random Access Memory (SRAM) is applied to store data for communication temporarily.

\subsubsection{HV control and monitor circuits}

A fixed $\mathrm{HV}$ is used for MCP, which can be adjusted from $-1600 \mathrm{~V}$ to $-2800 \mathrm{~V}$ according to the requirement of the spectrometer in different space environment. Moreover, there is one sweep $\mathrm{HV}$ for ESA ranging from $-2,800 \mathrm{~V}$ to $0 \mathrm{~V}$ and two sweep $\mathrm{HV}$ ranging from $-2,800 \mathrm{~V}$ to $2800 \mathrm{~V}$ for the inlet deflector. The changes of sweep HV can distinguish different energy ions and different incident directions because of structure of ESA and the inlet deflector. HV module (S9098/S9097, SITAEL) can output up to $\pm 3000 \mathrm{~V}$, which is stable and low-ripple. An Electrically Erasable Programmable Read Only Memory (EEPROM) chip is applied to store all parameters of HV step. To reduce single events effect (SEE), the TMR is applied to HV values of each step. Every HV step value are stored three times in the EEPROM chip. In the HV mode, the spectrometer will stay at each HV step for $100 \mathrm{~ms}$ or $1000 \mathrm{~ms}$ to collect the detector signals. Furthermore, the sweep HV step can be adjusted via instructions easily in order to detect ions with particular energy or direction. 
In order to monitor condition of the spectrometer, the Analog to Digital Converter (ADC) chips with a multiplexer are employed to collect the status information by the FPGA. 2 current values of LVPU, $7 \mathrm{HV}$ values and internal temperature of E-box are monitored. The power of the E-box is converted from the primary power supply through LVPU. If these monitor signal values exceed threshold, the FPGA will automatically cut off the HV supply to avoid irreversible damage.

\subsection{Software design}

The antifuse FPGA-based satellite onboard software flow design is shown in the Fig. 4. When LVPU is powered on, the system enters the self-test mode. However, the HVPU is power off by default. When FPGA software receives the HVPU power-on command from the satellite platform, it controls the power supply of the HVPU to boot. The fixed HV keeps a predetermined value while the sweep HV is configured by reading parameters from the EEPROM every $1 \mathrm{~s}$ by default. The configured HV is monitored at the same time. Once detecting abnormal HV value or receiving the HV stop command, the software will turn off the HVPU. If no abnormality occurs, software will continue to configure the next HV step value.

In addition to hardware design, software should also consider certain radiation-hardened methods, such as TMR method for all key registers, asynchronous signals synchronized before processing, and refreshing all external key registers of FPGA in about half an hour.

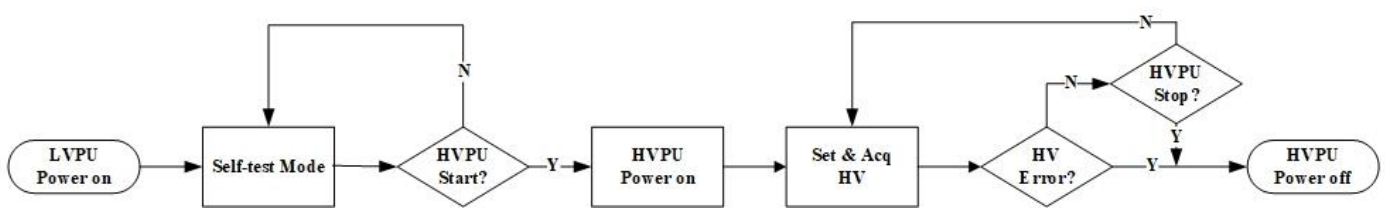

Fig. 4 Flow diagram of the satellite onboard software

\section{Results}

Fig. 5 (left) shows a photograph of flight model of the ion spectrometer instrument, after assembly of the sensor probe and the E-box. As shown in Fig. 5 (center), the anode plate of sensor probe consisting of 16 discrete anode parts. The board's edge is grounded to reduce noise. Fig. 5 (right) shows the core of readout electronics: DPU.

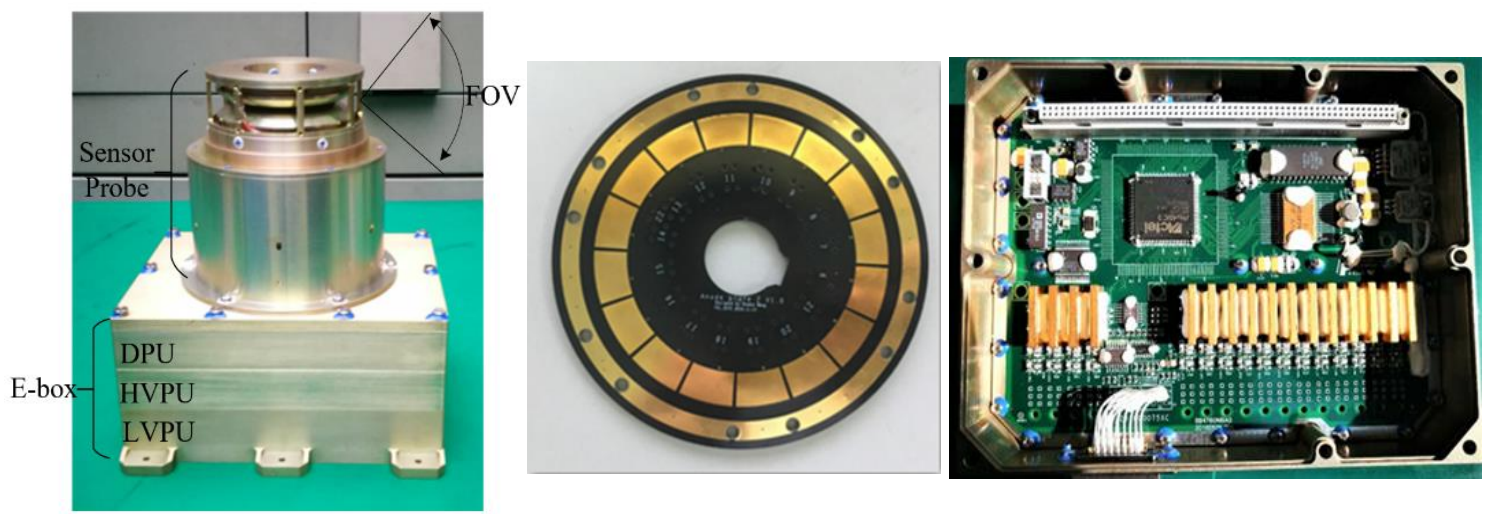

Fig. 5 Flight mode of the spectrometer (left); Anode plate (center); DPU in E-box (right) 


\subsection{Signal input and self-test circuit tests}

The threshold of A121 can be adjusted by a reference voltage. It is controlled by FPGA through a DAC; thus, the spectrometer can be adjusted remotely in the space. The relationship between the reference voltage and the threshold of input signal is shown in Fig. 6 (left).

The dynamic input range was also tested and analyzed by software. In the self-test mode, the threshold of the A121 was set to $90 \mathrm{fC}$ and the stimulus rate was set to $1 \mathrm{kHz}$. Then the FPGA counted A121 signals at different stimulus voltages. As shown in Fig. 6 (right), the electronics count $1 \mathrm{kHz}$ stably, when the input charge range was from $100 \mathrm{fC}$ to $350 \mathrm{fC}$. The counting rate increased with input charge beyond $350 \mathrm{fC}$ because of double pulsing effect [7].
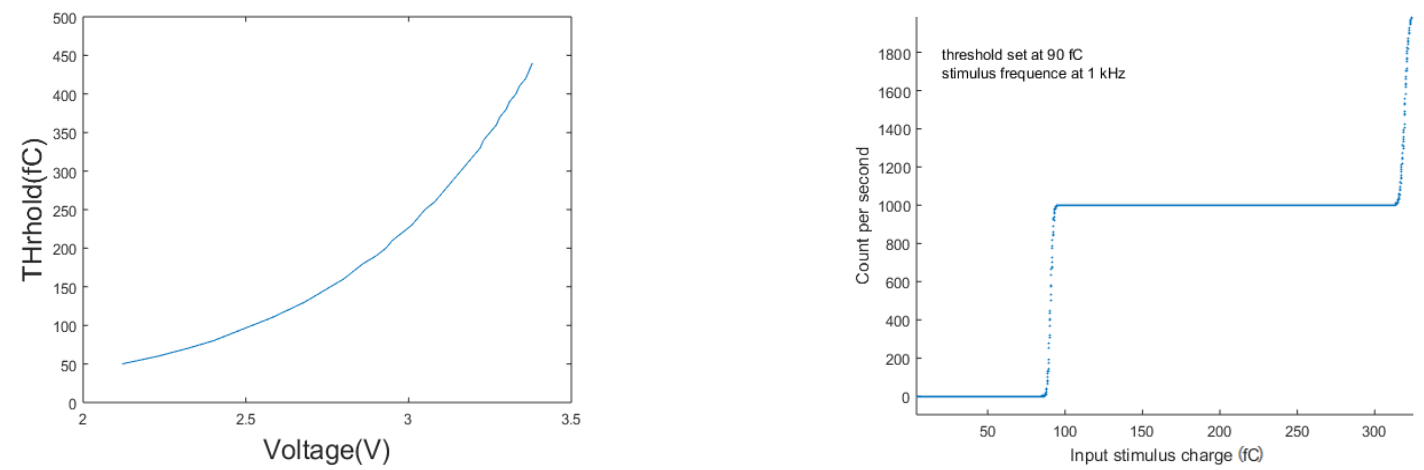

Fig. 6. Threshold of input signal vs control voltage (left); Counter record in different signal charge (right)

\subsection{System tests}

In order to verify the performance of the readout electronics, an experimental platform was set up (Fig. 7). The self-test and HV test were conducted. Test data are transferred to the computer via the RS422 interface for analysis. The analytical data in both modes is shown in Fig. 8. The count is around $1 \mathrm{kHz}$ in the self-test mode, and the count is 0 in the HV mode. The electronics work well in both modes, where the signal acquisition circuit has very low noise in HV mode. The max power of the system is $4.73 \mathrm{~W}$, with the max power of each unit as Fig. 9.
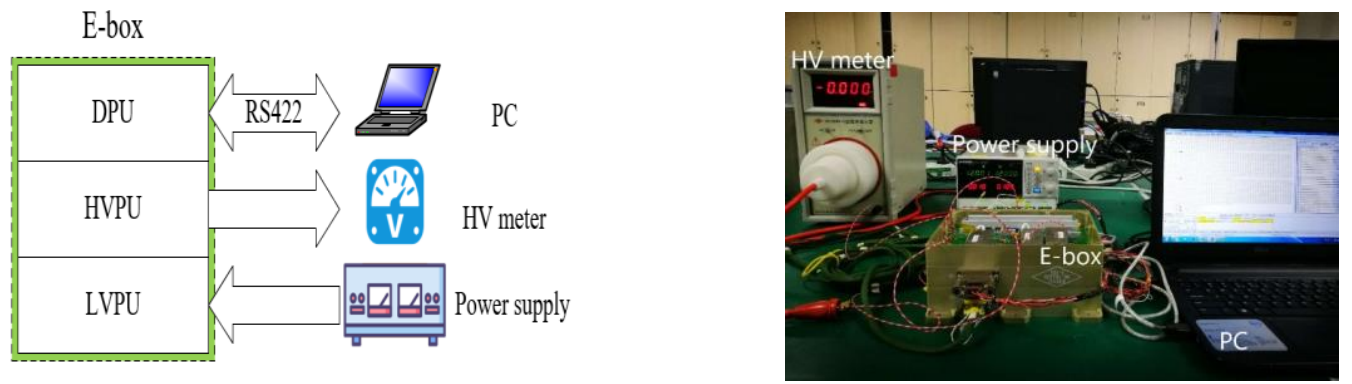

Fig. 7. Schematics of electronics system test (left); setup of electronics system test (right)

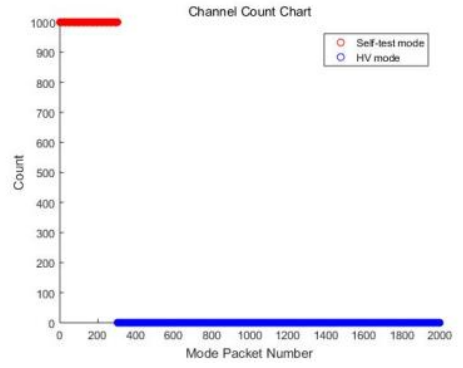

Fig. 8 Channel count in different mode

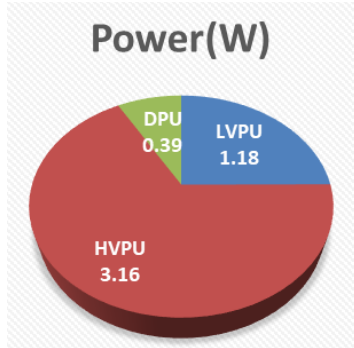

Fig.9 Max power of each unit 
To confirm the stability of data communication, the communication test between the readout electronics and the ground test module was ran for 10 days. Test results shows no bit error was detected. The Bit Error Rate (BER) was less than $2 \times 10^{-9}$.

\subsection{Environmental experiments}

A series of environmental experiments were carried out to verify the robustness of the flight mode of the spectrometer, including thermal-vacuum test, Electro Magnetic Compatibility (EMC) test, mechanics test, etc. In the thermal-vacuum tests, the spectrometer ran in vacuum with the pressure less than $1 \times 10^{-5} \mathrm{~Pa}, 7$ cycles with temperature range from $-55^{\circ} \mathrm{C}$ to $75^{\circ} \mathrm{C}$ in 7 days. The mechanics tests included mechanical impact test, random vibration test (total RMS acceleration of $16.7 \mathrm{~g}$ at vertical direction, $12.8 \mathrm{~g}$ at horizontal direction) and sinusoidal vibration test (total RMS acceleration of $10 \mathrm{~g}$ at both vertical direction and horizontal direction). The spectrometer operated stable in all tests, proving the readout electronics had been successfully designed.

\section{Conclusion}

The readout electronics has been successfully designed and produced for a low-energy ion spectrometer on a three-axis stabilized satellite in the near-earth space. According to requirements of the spectrometer, signal acquisition and self-test circuit based on A121, core control circuit based on antifuse FPGA, and HV control and monitor circuits are designed. Satellite onboard software using the TMR and timing refresh registers is also designed. The spectrometer had successfully operated stably in the system function tests and passed a series of environmental tests. The results show that the electronics meets the needs of the physical experiment of spectrometer.

\section{Acknowledgments}

We would like to thank Xiaoping Yang for help in the construction and environmental experiments in Shandong Institute of Space Electronic Technology. The work is supported by Natural Science Foundation of China (Grant No. 41327802) and Key Research Program of Frontier Science of Chinese Academy of Sciences (Number: QYZDB-SSW-DQC015).

\section{References}

[1] H. Reme, et al., The Cluster ion spectrometry (CIS) experiment. Space Sci. Rev. 79, 303-350 (1997).

[2] Paschmann, G. et al, PLASMA INSTRUMENT FOR AMPTE IRM. IEEE Transactions on Geoscience and Remote Sensing, v GE-23, n 3, p 262-266, (1985)

[3] R X Hu, et al., A low-energy ion spectrometer with half-space entrance for three-axis stabilized spacecraft. Sci China Tech Sci,2018,61

[4] McComas, D.J., et al., The Jovian Auroral Distributions Experiment (JADE) on the Juno Mission to Jupiter. 2017. 213(1): p. 547-643.

[5] A121 Charge Sensitive Preamplifier \& Discriminator. Amptek Inc., USA. http://amptek.com/products/a121-charge-sensitive-preamplifier-descriminator/

[6] L. Rockett, et al., Radiation Hardened FPGA Technology for Space Applications, IEEE Aerospace Conference, Big Sky, MT, 2007, pp. 1-7.

[7] D. Yang, et al., Readout electronics of a prototype spectrometer for measuring low-energy ions in solar wind plasma, Nucl. Sci. Tech. 27, 135 (2016). 